\title{
Comparison of the effect of rotenone and 1-methyl-4-phenyl-1,2,3,6-tetrahydropyridine on inducing chronic Parkinson's disease in mouse models
}

\author{
JING ZHANG $^{1 *}$, BOHAO SUN ${ }^{2 *}$, JIFENG YANG $^{1 *}$, ZHUO CHEN $^{1}$, \\ ZHENGZHENG $\mathrm{LI}^{3}$, NAN ZHANG ${ }^{3}$, HONGZHI LI ${ }^{1}$ and LUXI SHEN ${ }^{4}$
}

\begin{abstract}
${ }^{1}$ Zhejiang Provincial Key Laboratory of Medical Genetics, School of Laboratory Medicine and Life Sciences, Wenzhou Medical University, Wenzhou, Zhejiang 325035; ${ }^{2}$ Department of Pathology, Second Affiliated Hospital, School of Medicine, Zhejiang University, Hangzhou, Zhejiang 310009; ${ }^{3}$ Department of Internal Neurology, First Affiliated Hospital of Wenzhou Medical University, Wenzhou, Zhejiang 325035; ${ }^{4}$ Department of Internal Neurology, Beijing Friendship Hospital, Capital Medical University, Beijing 100050, P.R. China
\end{abstract}

Received August 17, 2021; Accepted December 16, 2021

DOI: 10.3892/mmr.2022.12607

\begin{abstract}
Animal models for Parkinson's disease (PD) are very useful in understanding the pathogenesis of PD and screening for new therapeutic approaches. The present study compared two commonly used neurotoxin-induced mouse models of chronic PD to guide model selection, explore the pathogenesis and mechanisms underlying PD and develop effective treatments. The chronic PD mouse models were established via treatment with rotenone or 1-methyl-4-phenyl-1,2,3,6tetrahydropyridine (MPTP) for 6 weeks. The effects of rotenone and MPTP in the mice were compared by assessing neurobehavior, neuropathology and mitochondrial function through the use of the pole, rotarod and open field tests, immunohistochemistry for tyrosine hydroxylase (TH), glial fibrillary acidic protein (GFAP), ionized calcium-binding adapter molecule 1 (Iba-1), neuronal nuclear antigen (NeuN) and (p)S129 $\alpha$-synuclein, immunofluorescence for GFAP, Iba-1 and NeuN, western blotting for TH, oxygen consumption, complex I enzyme activity. The locomotor activity, motor coordination and exploratory
\end{abstract}

Correspondence to: Dr Luxi Shen, Department of Internal Neurology, Beijing Friendship Hospital, Capital Medical University, 95 Yongan Road, Beijing 100050, P.R. China

E-mail: imaginary-diva@163.com

Professor Hongzhi Li, Zhejiang Provincial Key Laboratory of Medical Genetics, School of Laboratory Medicine and Life Sciences, Wenzhou Medical University, Northern Zhongshan Road, Chashan University Town, Wenzhou, Zhejiang 325035, P.R. China

E-mail:1hz@wmu.edu.cn

*Contributed equally

Key words: Parkinson's disease, 1-methyl-4-phenyl-1,2,3,6tetrahydropyridine, rotenone, mouse model, neurotoxin behavior in both rotenone and MPTP groups were significantly lower compared with the control group. However, behavioral tests were no significant differences between the two groups. In the MPTP group, the loss of dopaminergic (DA) neurons in the substantia nigra ( $\mathrm{SN}$ ) pars compacta, the reduction of the tyrosine hydroxylase content in the SN and striatum and the astrocyte proliferation and microglial activation in the SN were more significant compared with the rotenone group. Notably, mitochondrial-dependent oxygen consumption and complex I enzyme activity in the $\mathrm{SN}$ were significantly reduced in the rotenone group compared with the MPTP group. In addition, Lewy bodies were present only in SN neurons in the rotenone group. Although no significant differences in neurobehavior were observed between the two mouse models, the MPTP model reproduced the pathological features of PD more precisely in terms of the loss of DA neurons, decreased dopamine levels and neuroinflammation in the SN. On the other hand, the rotenone model was more suitable for studying the role of mitochondrial dysfunction (deficient complex I activity) and Lewy body formation in the SN, which is a characteristic pathological feature of PD. The results indicated that MPTP and rotenone PD models have advantages and disadvantages, therefore one or both should be selected based on the purpose of the study.

\section{Introduction}

Parkinson's disease (PD) is the second most common chronic progressive neurodegenerative disorder in the elderly population (1). The typical motor symptoms of PD include tremors, bradykinesia, muscle stiffness and postural instability (2). The typical pathological characteristics of PD include degeneration and loss of dopaminergic (DA) neurons in the substantia nigra (SN) pars compacta (SNpc) and the formation of Lewy bodies in residual neurons (3). Previous studies have indicated that aging, mitochondrial defects, oxidative stress, protein misfolding and aggregation and neuroinflammation are all involved in PD pathogenesis $(4,5)$. Defects in mitochondrial 
complex I activity and reduced ATP synthesis are routinely observed in PD (6).

In general, the etiology of PD is not clear. Genetic susceptibility and environmental exposures increase the risk of developing PD (7,8). As the majority of PD cases are sporadic, environmental factors may be also important (9). At present, there is no definitive cure, and the clinical diagnosis of PD is still based on the characteristic symptoms of motor dysfunction (10). Therefore, the etiology, pathology and therapeutic approaches for PD require detailed research for which the selection of appropriate animal models is essential.

Rotenone and 1-methyl-4-phenyl-1,2,3,6-tetrahydropyridine (MPTP) are common neurotoxins used to create animal PD models (11-15). Rotenone is a natural toxic substance extracted from plants in the Leguminosae family and is widely used in crop pest control and to eliminate fish from fish ponds (16). Epidemiological studies have demonstrated that the incidence of PD in individuals chronically exposed to rotenone is higher compared with that in the general population (17). Rotenone is liposoluble, allowing it to enter neurons directly without additional metabolic conversion or transporter-mediated movement, and inhibits mitochondrial complex I inducing neuronal degeneration $(18,19)$.

MPTP is non-toxic to neurons and freely enters the brain, where it is taken up by astrocytes, and is rapidly converted to the toxic metabolite 1-methyl-4-phenylpyridinium-iodide $\left(\mathrm{MPP}^{+}\right)$by monoamine oxidase. Once $\mathrm{MPP}^{+}$enters DA neurons via the dopamine transporter (DAT), a part of the $\mathrm{MPP}^{+}$is sequestered into synaptic vesicles by the vesicular monoamine transporter (20). In DA neurons, $\mathrm{MPP}^{+}$accumulates in the mitochondria, synaptic vesicles and cytoplasm; a part of the accumulated $\mathrm{MPP}^{+}$inhibits mitochondrial complex I (21). Although their mechanisms of action are not entirely understood, MPTP and rotenone both effectively induce PD-like symptoms (22).

Both rotenone and MPTP inhibit the activity of mitochondrial complex I, but they exhibit differences in the manner in which they enter neurons and their specific intracellular destinations (23). In the present study, the rotenone and MPTP-induced mouse PD models were produced using chronic exposure protocols, then compared based on aspects of neurobehavior, neuropathology and mitochondrial function. The aim of the present study was to provide a foundation to construct and select the most suitable PD animal model to explore the pathogenesis and mechanism of PD and develop more effective treatments.

\section{Materials and methods}

Experimental animals and groups. The animal research was approved by the Animal Care and Use Committee of Wenzhou Medical University (approval no. wydw2020-0840; Wenzhou, China). All animal experiments were performed in accordance with the Guide for the Care and Use of Laboratory Animals (NRC 2011) (http://oacu.od.nih. gov/regs/guide/guide_2011.pdf) and Guidelines for the ethical review of laboratory animal welfare People's Republic of China National Standard GB/T 35892-2018 (24). All animal experiments were performed by adequately trained researchers. Appropriate anesthetics (isoflurane, 3\% for induction; $1-1.5 \%$ for maintenance) were administrated to ease pain and suffering.

A total of 80 C57BL/6 male mice (age, 10-12 weeks; weight, 20-25 g) were purchased from Shanghai Shrek Experimental Animal Co., Ltd. All of the animal rooms were maintained at $20-25^{\circ} \mathrm{C}$ and humidity of $40-60 \%$, with a $12-\mathrm{h}$ light/dark cycle and free access to food and water. The mice were randomly divided into three groups. The MPTP group was injected intraperitoneally (IP), first with $250 \mathrm{mg} / \mathrm{kg}$ probenecid (MilliporeSigma), then $1 \mathrm{~h}$ later with $20 \mathrm{mg} / \mathrm{kg}$ MPTP (MilliporeSigma) dissolved in sterile physiological saline. The IP injections of probenecid and MPTP occurred twice a week for a total of 12 doses over 6 consecutive weeks (25). The rotenone group was administered $30 \mathrm{mg} / \mathrm{kg}$ rotenone suspended in $0.5 \%$ sodium carboxymethyl cellulose (CMC; MilliporeSigma) via daily administration by gavage for 6 consecutive weeks (26). The control group was administered the same volume (200-250 $\mu \mathrm{l})$ of $0.5 \% \mathrm{CMC}$ by gavage. After 6 weeks of administration, the survival rates were $100 \%$ (25/25) for the control group, $86.7 \%$ (26/30) for the rotenone group and 80\% (20/25) for the MPTP group. In our study, the control group that received $0.5 \% \mathrm{CMC}$ by gavage was compared with the control group that received $250 \mathrm{mg} / \mathrm{kg}$ probenecid via IP injection. The experiment was repeated three times and the results revealed no significant differences between these two control groups. Therefore, based on the previously published control data, the probenecid control group in the present study was not included.

Pole test. A total of five mice in each group were assessed for the degree of motor impairment using the pole test. Briefly, each mouse was placed at the top end of a $50 \mathrm{~cm}$ pole that had a radius of $4 \mathrm{~mm}$. The time each mouse needed to completely turn $180^{\circ}$ and climb down from the pole to the floor was recorded and averaged over three trials (27). Before the timed experiments, the mice in each group received 3 days of training with three trials per day.

Rotarod test. A total of five mice in each group were evaluated for motor coordination using the rotarod test. Briefly, mice were trained once a day for 3 days using a rotation speed of 10 revolutions per minute (rpm). The experiment was conducted on the fourth day using a rotation speed of $40 \mathrm{rpm}$. The duration each mouse remained on the rod with a maximum of $300 \mathrm{sec}$ was recorded (28).

Open field test. A total of five mice in each group were assessed using the open field test to evaluate their exploratory behavior. Each mouse was placed in an open field chamber $(40 \times 40 \mathrm{~cm})$ for $15 \mathrm{~min}$. The mouse movements were tracked, recorded and analyzed using the EthoVisionXT 11 software (Shanghai Jiliang Software Technology Co., Ltd.). The center zone was defined as a $20 \times 20 \mathrm{~cm}$ area in the center of the open field. The following data were analyzed: i) Total distance traveled; and ii) time in the center zone expressed as a percentage of the total distance traveled $(29,30)$.

Immunohistochemistry. Following the behavioral tests, five mice in each group were anesthetized with isoflurane (3\% induction; $1-1.5 \%$ maintenance) and perfused transcardially with $4 \%$ paraformaldehyde and post-fixed in PFA at $4^{\circ} \mathrm{C}$ overnight. The brain 
was removed and embedded in paraffin using routine protocols The immunohistochemical methods have been previously described (31). Tissue sections $(4-\mu \mathrm{m})$ mounted on glass microscope slides were deparaffinized in xylene and rehydrated in graded alcohols. Then the tissue sections were boiled for $15 \mathrm{~min}$ in $10 \mathrm{mM}$ citric acid buffer ( $\mathrm{pH}$ 6.0) for antigen retrieval. After the sections were cooled to room temperature (RT), they were incubated with $3 \% \mathrm{H}_{2} \mathrm{O}_{2}$ for $15 \mathrm{~min}$ at $37^{\circ} \mathrm{C}$ to block endogenous peroxidase activity. Then the sections were blocked with $5 \%$ goat serum (Scientific Phygene) for $45 \mathrm{~min}$ at room temperature, and incubated with primary antibodies, including mouse anti-mouse tyrosine hydroxylase (TH; 1:1,000; cat. no. 22941; Immunostar, Inc.), rabbit anti-mouse phosphorylated (p)S129 $\alpha$-synuclein (1:8,000; cat. no. ab51253; Abcam), rabbit anti-mouse glial fibrillary acidic protein (GFAP; 1:1,000; cat. no. ab68428; Abcam), rabbit anti-mouse ionized calcium-binding adapter molecule 1 (Iba-1; 1:1,000; cat. no. ab178847; Abcam) or mouse anti-mouse neuronal nuclear antigen (NeuN; 1:500; cat. no. MAB377; MilliporeSigma), overnight at $4^{\circ} \mathrm{C}$. Subsequently, the sections were incubated with secondary antibodies, including HRP-conjugated goat anti-mouse IgG antibody $(1: 1,000$; cat. no. ab6823; Abcam) or goat anti-rabbit IgG antibody (1:1,000; cat. no. ab6112; Abcam), for 90 min at RT. Finally, the substrate color was developed using a diaminobenzidine substrate kit (Abcam), and the sections were counterstained with hematoxylin (Beijing Solarbio Science \& Technology Co., Ltd.) for $1 \mathrm{~min}$ at room temperature. Image acquisition was performed using a light microscope (Nikon Corporation). Immunohistochemistry results were quantified using ImageJ software (version 1.8.0; National Institutes of Health). One field was randomly imaged from each of five different sections in each group to count the positive cells, and the average value was calculated.

Immunofluorescence. Three mice in each group were anesthetized with isoflurane as aforementioned and perfused transcardially with $4 \%$ paraformaldehyde to obtain brain tissue sections and post-fixed in PFA at $4^{\circ} \mathrm{C}$ overnight. The tissue sections were permeabilized with $0.5 \%$ Triton $\mathrm{X}-100$ for $20 \mathrm{~min}$ at room temperature and incubated with $5 \%$ goat serum for $45 \mathrm{~min}$ at room temperature. After washing in PBS, the sections were incubated overnight in rabbit anti-mouse GFAP antibody (1:1,000; cat. no. ab68428; Abcam), rabbit anti-mouse Iba-1 antibody (1:1,000; cat. no. ab178847; Abcam) or mouse anti-mouse NeuN antibody (1:500; cat. no. MAB377; MilliporeSigma) at $4^{\circ} \mathrm{C}$. Then the sections were incubated with secondary antibodies at $37^{\circ} \mathrm{C}$, Alexa Fluor 555-labeled donkey anti-rabbit IgG antibody (1:500; cat. no. A0453; Beyotime Institute of Biotechnology) or Alexa Fluor 488-labeled goat anti-mouse IgG antibody (1:500; cat. no. A0428; Beyotime Institute of Biotechnology), for $3 \mathrm{~h}$ and stained with DAPI (Beyotime Institute of Biotechnology) for $5 \mathrm{~min}$ at room temperature. Image acquisition was performed using a laser confocal microscope (Nikon Corporation). Immunofluorescent cells were quantified using ImageJ software. One field was randomly imaged from five different sections in each group to count the positive cells, and the average value was calculated.

Western blotting. A total of six mice in each group were anesthetized with isoflurane as aforementioned and perfused transcardially with PBS, the brain was removed and the SN tissue was isolated from both sides of the brain. The SN tissue was placed in RIPA lysis buffer (Beyotime Institute of Biotechnology), maintained for $30 \mathrm{~min}$ on ice and then centrifuged at $12,000 \mathrm{x}$ g for $2 \mathrm{~min}$ at $4^{\circ} \mathrm{C}$. The supernatant was collected from the homogenized tissue after centrifugation. The protein concentration was determined using a BCA reagent (Beyotime Institute of Biotechnology) with bovine serum albumin as the protein standard. Proteins (20 $\mu \mathrm{g} / \mathrm{lane})$ were separated on $10 \%$ SDS-PAGE and transferred to polyvinylidene fluoride membranes for immunoblotting. After that, the membrane was blocked using 5\% skim milk powder at room temperature for $90 \mathrm{~min}$, The membranes were incubated overnight with primary antibodies, including rabbit anti-mouse TH (1:1,000; cat. no. AF2185; Beyotime Institute of Biotechnology) and mouse anti-mouse GAPDH (1:5,000; cat. no. 60004-lg; Wuhan Sanying Biotechnology), at $4^{\circ} \mathrm{C}$. Subsequently, the membranes were incubated at RT with secondary antibodies, including HRP-labeled goat anti-mouse IgG antibody (1:1,000; cat. no. A0216; Beyotime Institute of Biotechnology) or HRP-labeled goat anti-rabbit IgG antibody (1:1,000; cat. no. A0208; Beyotime Institute of Biotechnology), for $90 \mathrm{~min}$. The blots were developed utilizing electrochemiluminescence reagent (Beyotime Institute of Biotechnology), and detection was accomplished using the ChemiDoc MP Imaging System (Bio-Rad Laboratories, Inc.). The gray values of the bands were scanned and analyzed using Image J software.

Oxygen consumption measurement. A total of three mice in each group were anesthetized with isoflurane as aforementioned and perfused transcardially with PBS, and the SN tissue on both sides of the dissected brain was removed. Briefly, the SN tissue was homogenized in ice-cold buffer A \{pH 7.4, $5 \mathrm{mM} \mathrm{KCl}, 5.8 \mathrm{mM} \mathrm{NaCl}, 2 \mathrm{mM} \mathrm{MgCl}_{2}, 0.75 \mathrm{mM}$ $\mathrm{CaCl}_{2}, 137 \mathrm{mM}$ sucrose and $10 \mathrm{mM}$ 2-[4-(2-hydroxyethyl) piperazin-1-yl)] ethanesulfonic acid (HEPES)\} and centrifuged for $5 \mathrm{~min}$ at $1,000 \mathrm{x}$ at $4^{\circ} \mathrm{C}$ (32). The supernatant was removed and further centrifuged for $2 \mathrm{~min}$ at $15,000 \mathrm{xg}$ at $4{ }^{\circ} \mathrm{C}$. The resulting pellets were resuspended in Buffer $\mathrm{B}$ [20 mM HEPES (pH 7.1), $250 \mathrm{mM}$ sucrose, $2 \mathrm{mM} \mathrm{KH}_{2} \mathrm{PO}_{4}$, $10 \mathrm{mM} \mathrm{MgCl}$ and $1 \mathrm{mM} \mathrm{ADP]}$. The mitochondrial protein concentration was determined using a BCA kit (Beyotime Institute of Biotechnology). Subsequently, $80 \mu \mathrm{g}$ of the mitochondrial solution was transferred to an Oxygraph-2k chamber (O2k; Oroboros Instruments $\mathrm{GmbH})$. Subsequently, $5 \mathrm{mM}$ malic acid, $5 \mathrm{mM}$ glutamic acid and $5 \mathrm{mM}$ succinate were added to measure the total oxygen consumption of mitochondrial complexes I and II. Oligomycin $(2.5 \mu \mathrm{g} / \mathrm{ml})$ was added to record the level of oxygen consumption uncoupled to ATP synthase. Subsequently, $0.1 \mu \mathrm{M}$ carbonyl cyanide-ptrifluoromethoxyphenylhydrazone (FCCP) was added to determine the maximum respiration rate. All measurements were performed according to the manufacturer's instructions of the O2k chamber and the oxygen flux per mass was recorded using Oroboros DatLab Software 5.2.1.51 (Oroboros Instruments $\mathrm{GmbH}$ ).

Mitochondrial respiratory chain complex I enzyme activity. A total of five mice in each group were anesthetized with isoflurane as aforementioned and perfused transcardially with PBS, and the SN tissue was removed on both sides of the dissected 
brain. Briefly, the tissue was homogenized in ice-cold tissue lysis buffer (Beijing Solarbio Science \& Technology Co., Ltd.) and centrifuged for $5 \mathrm{~min}$ at $1,000 \mathrm{x} \mathrm{g}$ at $4^{\circ} \mathrm{C}$. The supernatant was removed and further centrifuged for $10 \mathrm{~min}$ at $12,000 \mathrm{x} \mathrm{g}$ at $4^{\circ} \mathrm{C}$. The resulting pellets were resuspended in Buffer $\mathrm{A}$ as aforementioned (33). The mitochondria were frozen and thawed three times using liquid nitrogen, and the mitochondrial protein concentration was measured using a BCA kit (Thermo Fisher Scientific, Inc.).

The mitochondrial respiratory chain complex I enzyme and citrate synthase activity in the $\mathrm{SN}$ were detected using a U-3900 spectrophotometer (Hitachi, Ltd.). The reaction mixture consisting of $0.5 \mathrm{M} \mathrm{NaN}, 10 \mathrm{mM}$ nicotinamide adenine dinucleotide (NADH) and $4 \mu \mathrm{g}$ mitochondrial protein, was incubated at $37^{\circ} \mathrm{C}$ for $2 \mathrm{~min}$. The oxidation rate of $\mathrm{NADH}$ was measured by adding $6 \mathrm{mM}$ ubiquinone and assessed at $340 \mathrm{~nm}$. Subsequently, the reaction mixture including $1 \mathrm{mM}$ 5,5'-dithiobis (2-nitrobenzoic acid), $10 \mathrm{mM}$ acetyl coenzyme A and $4 \mu \mathrm{g}$ mitochondrial protein, was incubated at $37^{\circ} \mathrm{C}$ for $2 \mathrm{~min}$. The rate of citric acid generation was measured by adding oxaloacetic acid and assessed at $412 \mathrm{~nm}$. The enzyme activity of mitochondrial complex I was normalized with the activity of citric acid synthase. Enzyme activity measurements were repeated three times independently.

Statistical analysis. Data are presented as mean \pm standard deviation. P-values were calculated using SPSS 22.0 software (IBM Corp.). Unpaired Student's t-tests were used to compare data between two groups. Comparisons among three groups were performed using one-way ANOVA. First, the homogeneity of variance was tested. If equal variances were observed, then the P-values were calculated using Tukey's post hoc test. Otherwise, the P-values were calculated using Tamhane's T2 test. $\mathrm{P}<0.05$ was considered to indicate a statistically significant difference.

\section{Results}

Results of behavioral tests are significantly altered in the rotenone and MPTP mouse PD models compared with the control, but no significant difference is observed between the models. Behavioral assessments were conducted to investigate the motor function of the rotenone and MPTP models, including the pole, rotarod and open field tests, to evaluate changes in locomotion, motor coordination and exploratory behavior, respectively. In the pole test (Fig. 1A and B), rotenone and MPTP treatments significantly extended the time required to turn $180^{\circ}$ and the total time needed for the mouse to reach the floor (locomotion activity time) compared with the control group (both $\mathrm{P}<0.01$ ). There was no significant difference between the MPTP and rotenone groups. The images of the pole test are presented in Fig. S1. In the rotarod test (Fig. 1C), the fall latencies for the rotenone and MPTP groups were significantly shorter compared with the control group (both $\mathrm{P}<0.01$ ). By contrast, there was no significant difference between the MPTP and rotenone groups. The images of the rotarod test are presented in Fig. S2.

In the open field test (Fig. 1D-F), the total distance traveled was significantly shorter and the ratio of the central distance to the total distance was smaller in the rotenone and MPTP groups compared with the control group (all $\mathrm{P}<0.01)$. However, there was no significant difference between the MPTP and rotenone groups. These results indicated that the rotenone and MPTP-treated mice exhibited a significant decrease in motor function and exploratory behavior, but there was no significant difference between the two experimental groups. After completion of the neurobehavioral tests, the survival rates for the control, rotenone and MPTP groups were 100, 86.7 and $80 \%$, respectively.

Degeneration and death of DA neurons in the SNpc of the rotenone and MPTP mouse PD models are significantly increased compared with the control, especially in the MPTP model. An important pathological process in the brains of patients with PD is the degeneration of DA neurons in the SNpc (34). TH, the rate-limiting enzyme in dopamine synthesis, is widely recognized as a specific marker to evaluate the survival status of DA neurons (35). DA neuronal survival was evaluated by determining the number of $\mathrm{TH}$-positive neurons in the SNpc. The distribution of neurons in the positively stained region appeared to be in the shape of an inverted, elongated comma (Fig. 2A). To detect the degree of DA neuronal damage in the SNpc of the rotenone and MPTP models, the number of surviving DA neurons in the ventral tegmental area (VTA) and the SNpc were quantitatively compared in each group. There was no significant difference in the numbers of DA neurons in the VTA among all groups (Fig. 2B). Furthermore, the results demonstrated that exposure to rotenone and MPTP resulted in no detectable damage in the VTA (Fig. 2B). However, the number of surviving neurons in the SNpc was significantly lower in the rotenone and MPTP groups compared with the control group ( $\mathrm{P}<0.001$; Fig. $2 \mathrm{C}$ ). In addition, the number of surviving neurons was significantly lower in the MPTP group compared with the rotenone group $(\mathrm{P}<0.01$; Fig. 2C). No significant difference in the number of surviving DA neurons in SNpc and VTA (Fig. S3A-C) were revealed between the control group of $0.5 \% \mathrm{CMC}$ administered via gavage and the control group of $250 \mathrm{mg} / \mathrm{kg}$ probenecid administered via IP injection.

In PD, degeneration and loss of DA neurons in the SN results in decreased TH expression (36). To further explore the degree of damage to DA neurons in the $\mathrm{SN}$ in the rotenone and MPTP models, the expression level of TH in the SN was assessed using western blotting. TH expression in the SN (Fig. 2D and E) was significantly lower in the rotenone and MPTP groups compared with the control group (both $\mathrm{P}<0.01$ ). Notably, the TH expression was considerably lower in the MPTP group compared with that of the rotenone group $(\mathrm{P}<0.01)$. These results revealed that rotenone and MPTP caused the degeneration and death of DA neurons in the $\mathrm{SNpc}$ and indicated that the toxic effects of MPTP were more pronounced.

TH protein content in the striatum of the rotenone and MPTP mouse PD models is significantly lower compared with the control, especially in the MPTP model. The TH protein content in the striatum is considered to be an indirect indication of the striatal dopamine content (37). The TH protein expression level in the striatum was detected using immunohistochemistry. The optical density of TH-positive 
A

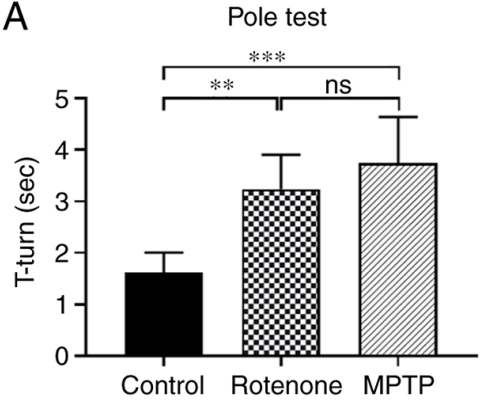

D

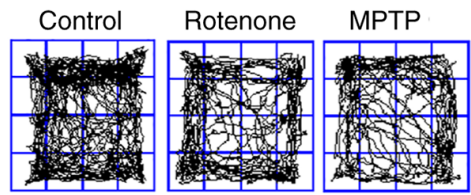

B

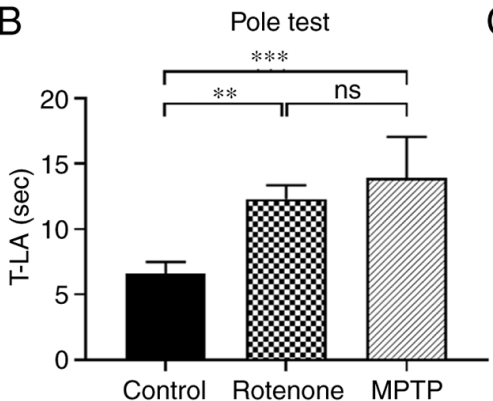

E

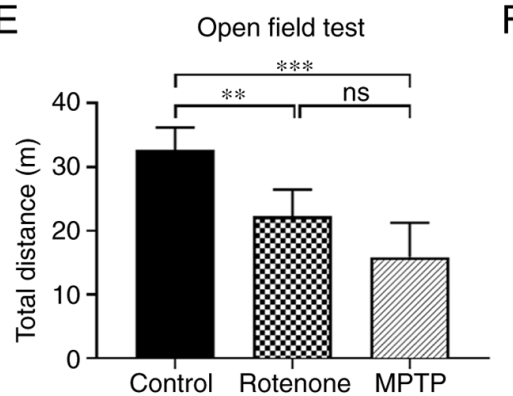

C

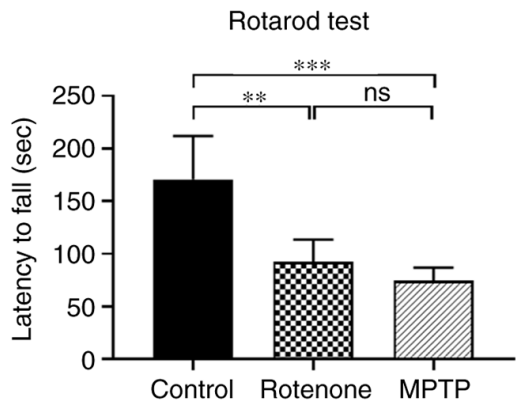

F

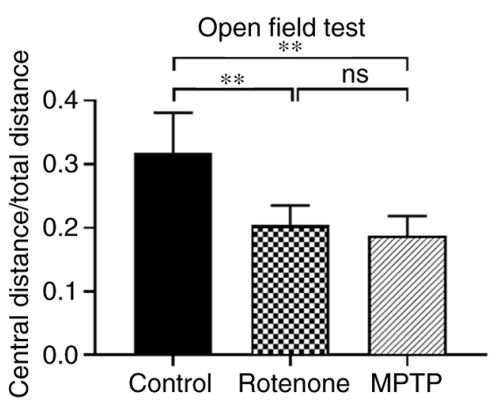

Figure 1. Neurobehavioral tests. (A) Time taken for mice to turn $180^{\circ}$. (B) Total time taken for mice to reach the floor. (C) Time the mouse remained on the rod in the rotarod test. (D) A total of 15 min movement track segments of open field exploration by the mice. (E) Total distance traveled in the open field test. (F) Ratio of central distance to the total distance in the open field test. ${ }^{* *} \mathrm{P}<0.01,{ }^{* * *} \mathrm{P}<0.001$. ns, no significant difference; T-turn, time to turn; T-LA, time of locomotion activity; MPTP, 1-methyl-4-phenyl-1,2,3,6-tetrahydropyridine.

A

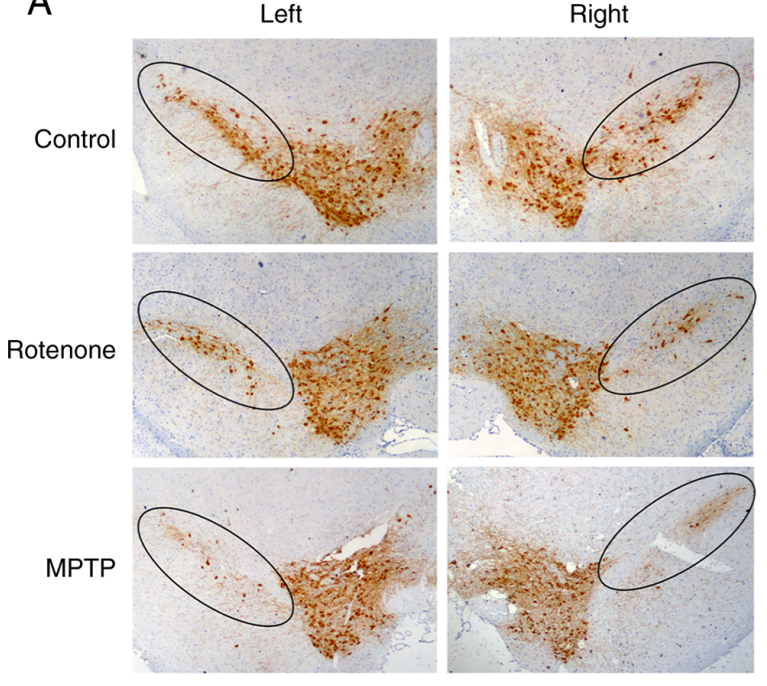

B
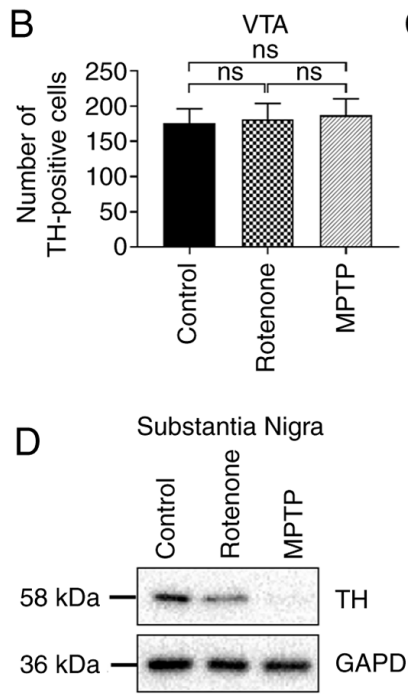

C SNpc

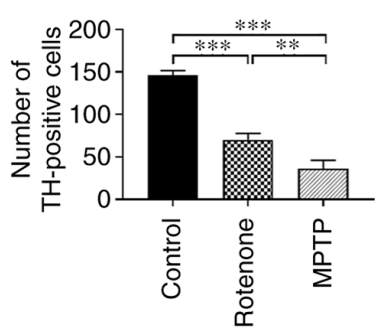

$\mathrm{E}$

Substantia Nigra

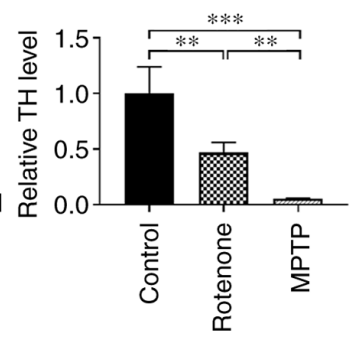

Figure 2. Neuronal degeneration and death in the SNpc in the rotenone and MPTP mouse Parkinson's disease models. (A) Immunohistochemical staining for $\mathrm{TH}$ in the SNpc. TH protein is expressed in the cytoplasmic regions of dopaminergic neurons; the inner circle area represents the SNpc; the outer circle area in brown represents the VTA. Magnification, x40. Quantitative analysis of the number of surviving neurons in the (B) VTA and (C) SNpc. (D) Expression level of TH protein in the SN was detected using western blotting; GAPDH was used as the internal reference standard. (E) Quantitative analysis of TH expression in the $\mathrm{SN} .{ }^{* *} \mathrm{P}<0.01,{ }^{* * *} \mathrm{P}<0.001$. ns, no significant difference; VTA, ventral tegmental area; SN, substantia nigra; SNpc, SN pars compacta; MPTP, 1-methyl-4-phenyl-1,2,3,6-tetrahydropyridine; TH, tyrosine hydroxylase.

staining in the striatum (Fig. 3A and B) was significantly lower in the rotenone and MPTP groups compared with the control group (both $\mathrm{P}<0.01$ ), and the staining was significantly lower in the MPTP group compared with the rotenone group
$(\mathrm{P}<0.05)$. No significant difference in the content of dopamine in the striatum (Fig. S3D and E) was revealed between the control group of $0.5 \% \mathrm{CMC}$ and that of $250 \mathrm{mg} / \mathrm{kg}$ probenecid. Furthermore, the expression level of TH protein in the 


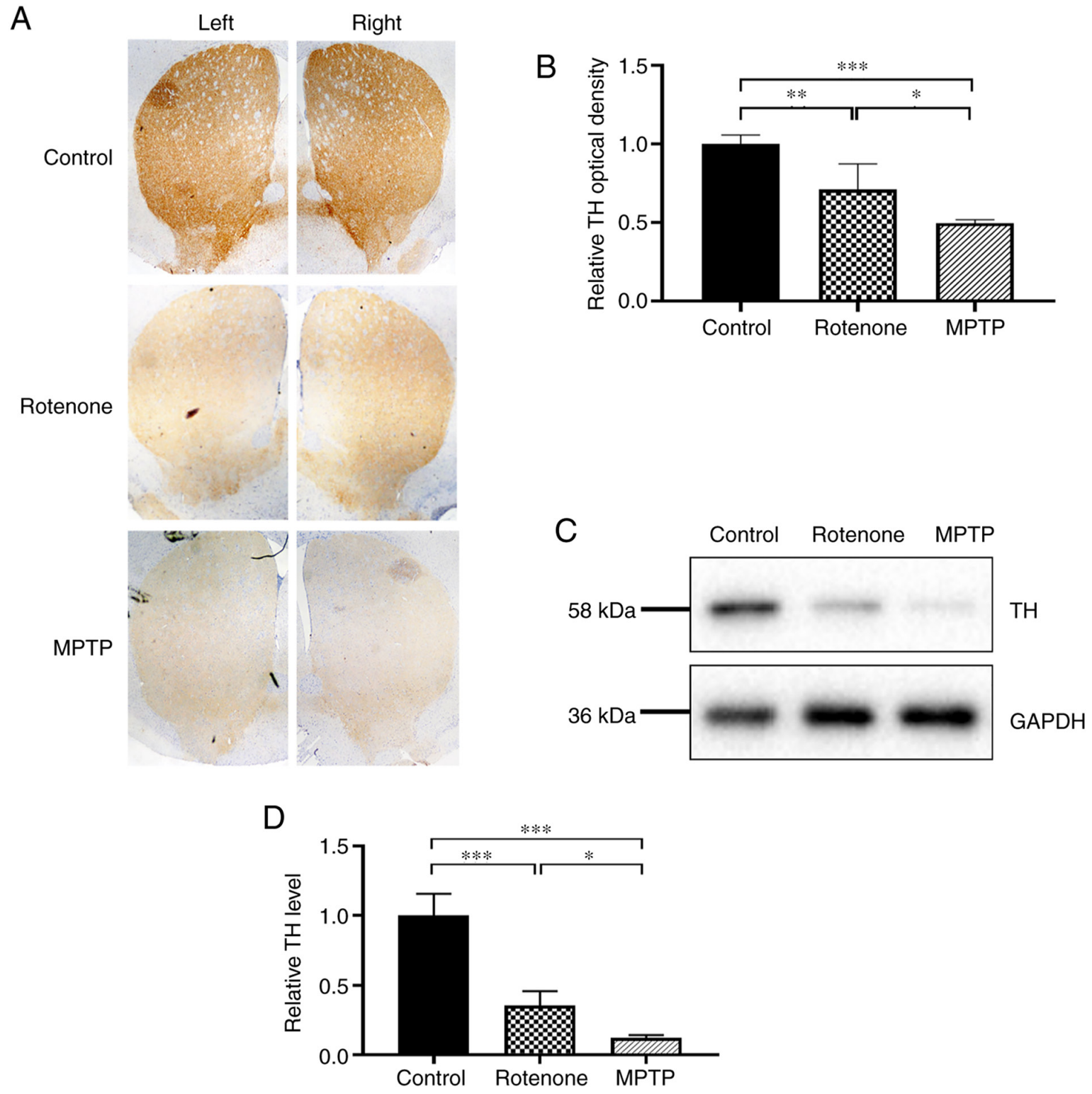

Figure 3. TH expression in the striatum of the rotenone and MPTP mouse Parkinson's disease models. (A) Immunohistochemical staining for TH in the striatum. Magnification, x40. (B) Quantitative analysis of TH optical density in the striatum. (C) Expression level of TH protein was detected using western blotting; GAPDH was used as the internal reference standard. (D) Quantitative analysis of TH expression. ${ }^{*} \mathrm{P}<0.05,{ }^{* *} \mathrm{P}<0.01,{ }^{* * *} \mathrm{P}<0.001$. TH, tyrosine hydroxylase; MPTP, 1-methyl-4-phenyl-1,2,3,6-tetrahydropyridine.

striatum was detected using western blotting. TH expression in the striatum (Fig. 3C and D) was significantly lower in the rotenone and MPTP groups compared with the control group (both $\mathrm{P}<0.001$ ), and it was significantly lower in the MPTP group compared in the rotenone group $(\mathrm{P}<0.05)$. These results demonstrated that both rotenone and MPTP treatments significantly reduced the protein level of $\mathrm{TH}$ in the striatum and indicated more pronounced toxic effects for MPTP.

Lewy bodies appear in SNpc neurons in the rotenone model but not the MPTP model. Another characteristic pathological change observed in PD is the formation of Lewy bodies in the residual SNpc neurons. Lewy bodies can activate surrounding microglia, promote the release of inflammatory mediators, and aggravate neuronal injury (38). A small fraction of $\alpha$-synuclein is phosphorylated at the S129 site $(<4 \%)$ in healthy brains, but it has been observed to be accumulated ( $90 \%)$ in the brains of patients with PD (39). Therefore, pS129 $\alpha$-synuclein was used as a marker to evaluate the pathological changes associated with $\alpha$-synuclein. Lewy bodies were observed in the rotenone group using immunohistochemical staining for $\mathrm{pS} 129 \alpha$-synuclein, but not in the control or MPTP groups (Fig. 4A and B). These results demonstrated that, compared with MPTP, rotenone could induce the formation of Lewy bodies in the residual neurons in the SNpc and provided a more accurate simulation of the pathological processes that occur in patients with PD.

MPTP and rotenone induce glial activation and damaged neurons. The importance of neuroinflammation in mediating the progressive death of DA neurons in PD has been recognized. Astrocyte and microglial activation in the central nervous system provides evidence of the existence of inflammation in PD (40,41). GFAP and Iba-1 are molecular markers of activated astrocytes and microglia, respectively, and their 
A

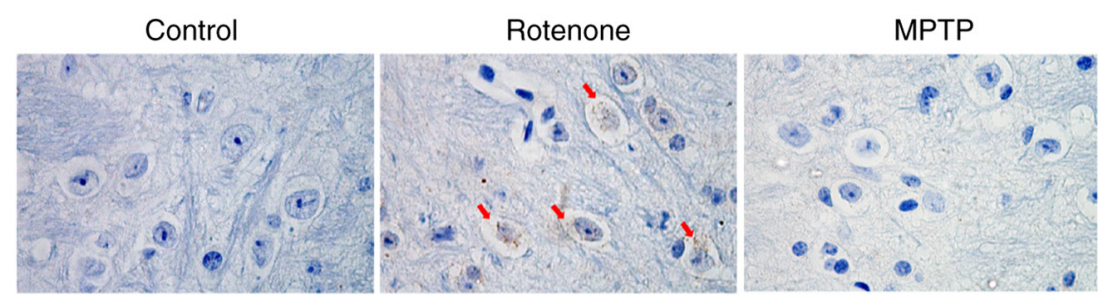

B

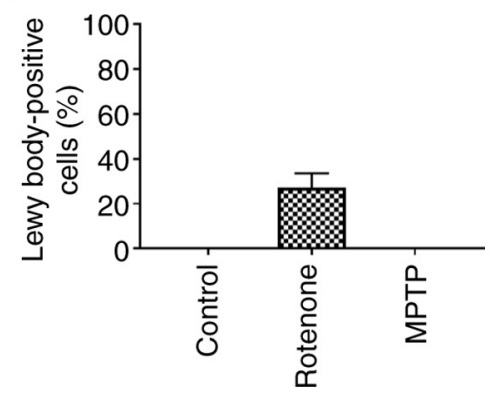

Figure 4. Lewy body formation in substantia nigra pars compacta neurons in rotenone-treated mice. (A) Phosphorylated S129 $\alpha$-synuclein protein expression in the cytoplasmic region of dopaminergic neurons indicated the presence of Lewy bodies. The grainy-appearing brown bodies marked by the red arrows are Lewy bodies. Magnification, x1,000. (B) Quantitative analysis of the proportion of Lewy body-positive cells. MPTP, 1-methyl-4-phenyl-1,2,3,6-tetrahydropyridine.

activation is closely associated with injury or inflammation in the brain (42). To reveal the activation of astrocytes and microglia in the SN, GFAP and Iba-1 in the SN were detected using immunofluorescence and immunohistochemistry. The number of GFAP-positive cells in the SN (Fig. 5A, D, G and H) was significantly increased in the rotenone and MPTP groups compared with the control group (all $\mathrm{P}<0.01$ ). Also, the number was significantly higher in the MPTP group compared with the rotenone group (both $\mathrm{P}<0.01$ ). The number of Iba-1-positive cells in the SN (Fig. 5B, E, G and I) was significantly increased in the rotenone and MPTP groups compared with the control group (all $\mathrm{P}<0.01$ ). The number was significantly higher in the MPTP group compared with in the rotenone group (both $\mathrm{P}<0.01)$. Thus, these results demonstrated that treatment with rotenone and MPTP could enhance the activation of astrocytes and microglia in the SN and revealed that MPTP exhibited more pronounced toxic effects.

$\mathrm{NeuN}$ is expressed in the nucleus of the majority of neurons in the vertebrate nervous system (43). It is one of the most commonly used markers for neurons. Thus, the present study examined NeuN expression to determine whether there was a similar effect as observed with TH expression. The number of NeuN-positive cells in the SN (Fig. 5C, F, G and J) was significantly decreased in the rotenone and MPTP groups compared with the control group (all $\mathrm{P}<0.01$ ). Also, the number was significantly decreased in the MPTP group compared with the rotenone group (both $\mathrm{P}<0.05$ ). These observations suggested that there was neuronal loss in addition to decreased $\mathrm{TH}$ expression in the surviving neurons in the rotenone and MPTP mouse PD models. The results also suggested that MPTP exhibited more toxic effects compared with rotenone.

Rotenone and MPTP decreased mitochondrial-dependent oxygen consumption and complex I enzyme activity compared with the control, especially in the rotenone model. Rotenone and MPTP are direct and indirect inhibitors of mitochondrial complex I, respectively, and the underlying mechanisms are different (23). Therefore, the present study determined the oxygen consumption and complex I activity to assess the effects of rotenone and MPTP on mitochondrial function.

The mitochondrial oxygen consumption level in the SN was detected using O2k. In Fig. 6A, complex I and complex II $(\mathrm{CI}+\mathrm{CII})$ represented the total oxygen consumption of mitochondrial complexes I and II, which were significantly lower in the rotenone and MPTP groups compared with the control group (both $\mathrm{P}<0.001$ ). Also, the total oxygen consumption for the rotenone group was considerably lower compared with the MPTP group $(\mathrm{P}<0.05)$. Oxygen consumption after oligomycin treatment (Oligo), an inhibitor of complex V (ATP synthase) of the electron transport chain, was significantly lower in the rotenone and MPTP groups compared with the control group (both $\mathrm{P}<0.05$ ). The oligo was not different between the rotenone and MPTP groups. FCCP (oxygen consumption after FCCP treatment) represents the maximum oxygen consumption after uncoupling the electron transport chain, which was significantly lower in the rotenone and MPTP groups compared with the control group (both $\mathrm{P}<0.001$ ). Also, the FCCP in the rotenone group was considerably lower compared with the MPTP group $(\mathrm{P}<0.05)$. These results indicated that both rotenone and MPTP significantly altered the oxygen consumption of mitochondria in the SN and suggested that rotenone had a more pronounced effect on oxygen consumption.

To examine whether the coupling of substrate oxidation to ATP production was impaired, the present study assessed the mitochondrial coupling efficiency using the respiratory control ratio (RCR) and leak control ratio (LCR). For the RCR that was defined as the $(\mathrm{CI}+\mathrm{CII}) /$ oligo ratio (Fig. 6B), the lower the value, the lower the efficiency of electron transport chain coupling with oxidative phosphorylation. The RCR values decreased significantly in the rotenone and MPTP groups compared with the control group (both $\mathrm{P}<0.01$ ). The RCR was not significantly different between the rotenone and MPTP groups. For the LCR, which was defined as the oligo/FCCP ratio (Fig. 6C), the larger the value the lower the coupling efficiency of mitochondria. The LCR was significantly higher in the rotenone and MPTP groups compared with in the control group (both $\mathrm{P}<0.01$ ). Furthermore, the LCR was considerably higher in the rotenone group compared with the MPTP group $(\mathrm{P}<0.05)$. These results revealed that both rotenone and MPTP decreased the coupling efficiency of mitochondria in the $\mathrm{SN}$ and suggested that the effects of rotenone were more pronounced.

Mitochondrial complex I enzyme activity was measured as the rate of NADH oxidation (Fig. 6D). This assessment was based on the characteristic that complex I (NADH dehydrogenase) catalyzes NADH dehydrogenation to $\mathrm{NAD}^{+}$when measured at $340 \mathrm{~nm}$ and was normalized with the activity of citric acid synthase (44). The activity of mitochondrial 
A

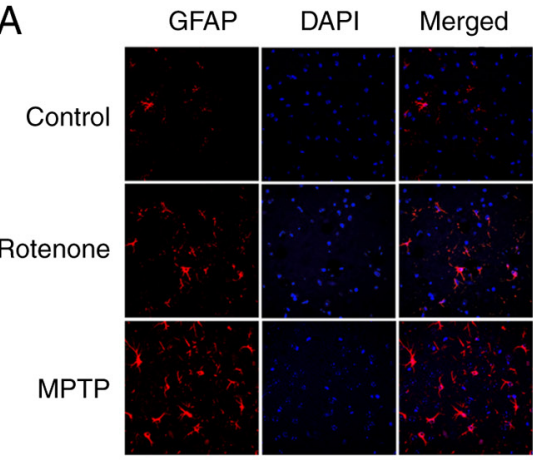

$\mathrm{D}$

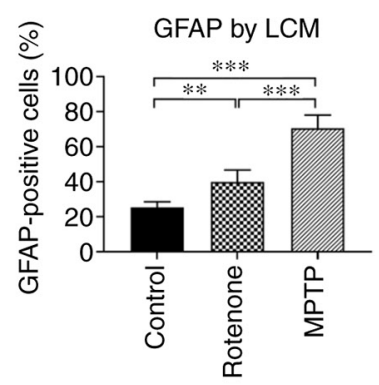

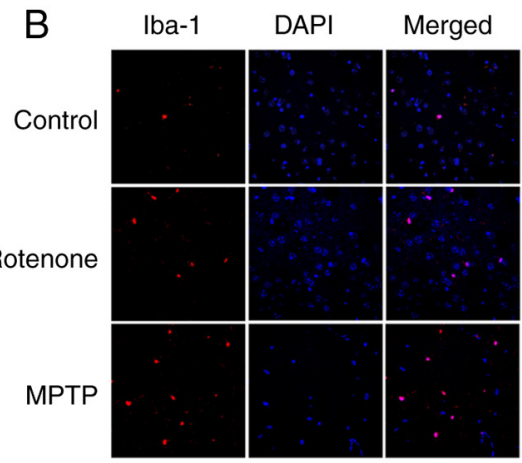

E

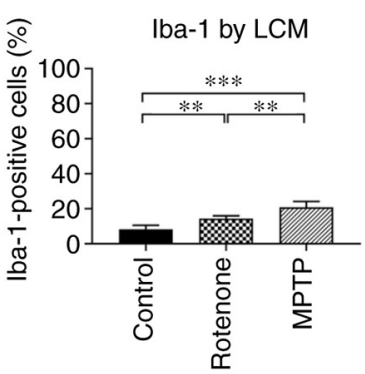

C

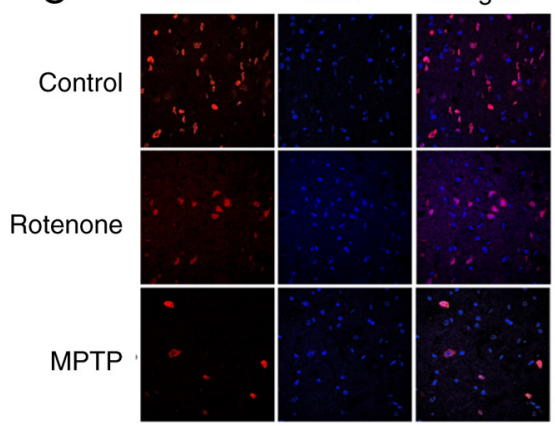

$\mathrm{F}$

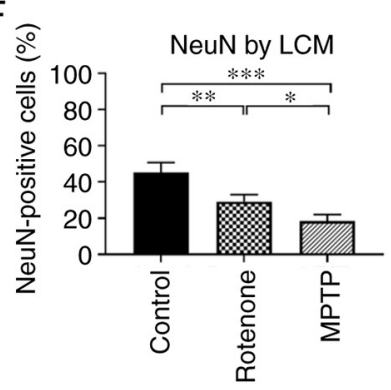

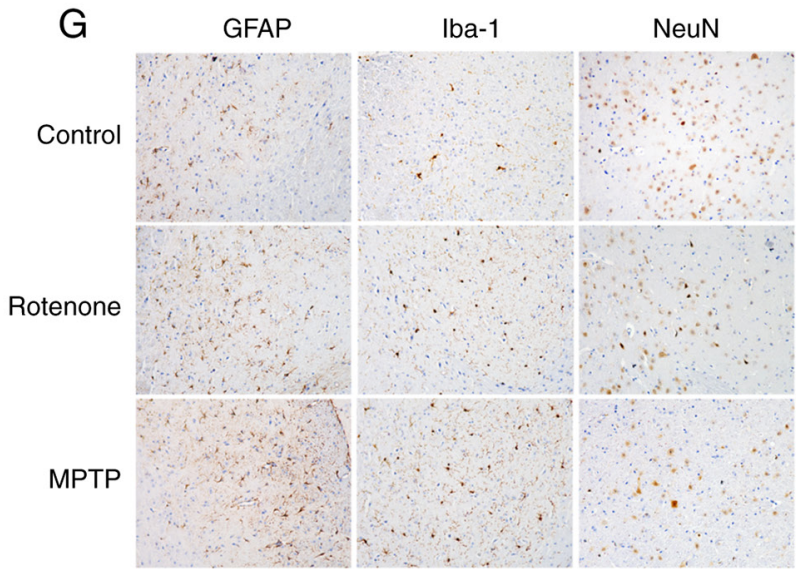
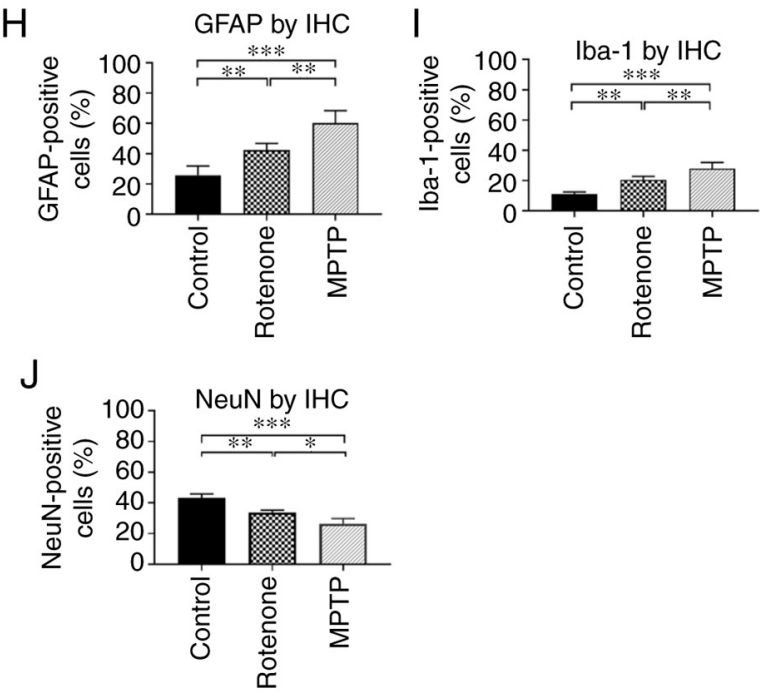

Figure 5. Number of astrocytes, microglia and neurons in the substantia nigra of the rotenone and MPTP mouse Parkinson's disease models. (A) Number of astrocytes (GFAP-positive) was measured using LCM. GFAP protein (red) is expressed in the cytoplasmic regions of the astrocytes. DAPI (blue) indicates the nucleus. Magnification, Annexin V. (B) Number of microglia (Iba-1-positive) was detected using LCM. Iba-1 protein (red) is expressed in the cytoplasmic regions of the microglia. DAPI (blue) indicates the nucleus. Magnification, Annexin V. (C) Number of NeuN-positive cells was detected using LCM. NeuN protein was expressed in the nuclei of the majority of neurons. DAPI (blue) indicates the nucleus. Magnification, 600x. (D) Quantitative analysis of the proportion of astrocytes (GFAP-positive). (E) Quantitative analysis of the proportion of microglia (Iba-1-positive). (F) Quantitative analysis of the proportion of NeuN-positive cells. (G) Number of astrocytes (GFAP-positive), microglia (Iba-1-positive) and NeuN-positive cells were detected using IHC. GFAP, Iba-1 and NeuN proteins are expressed in the cytoplasmic regions of astrocytes, microglia and the nucleus of the majority of neurons, respectively. Scale bar, $100 \mu \mathrm{m}$. Quantitative analysis of the proportion of $(\mathrm{H})$ astrocytes, (I) microglia and (J) NeuN-positive cells. ${ }^{*} \mathrm{P}<0.05,{ }^{* *} \mathrm{P}<0.01,{ }^{* * *} \mathrm{P}<0.001$. LCM, laser confocal microscopy; IHC, immunohistochemistry; NeuN, neuronal nuclear antigen; GFAP, glial fibrillary acidic protein; MPTP, 1-methyl-4-phenyl-1,2,3,6-tetrahydropyridine; Iba-1 ionized calcium-binding adapter molecule 1.

complex I enzyme was significantly lower in the rotenone and MPTP groups compared with the control group (both $\mathrm{P}<0.001)$, and the activity in the rotenone group was considerably lower compared with the MPTP group $(\mathrm{P}<0.01)$. These results demonstrated that both rotenone and MPTP could significantly inhibit mitochondrial complex I enzyme activity and indicated that the effects produced by rotenone were more important.

\section{Discussion}

The incidence of PD may result from genetic and environmental factors and is divided into two forms, familial and sporadic. Overall, $~ 95 \%$ of PD cases are sporadic and may be associated with environmental factors (45). Currently, animal models of PD primarily include neurotoxin-based animal models and genetic animal models, each of which have 
A

A

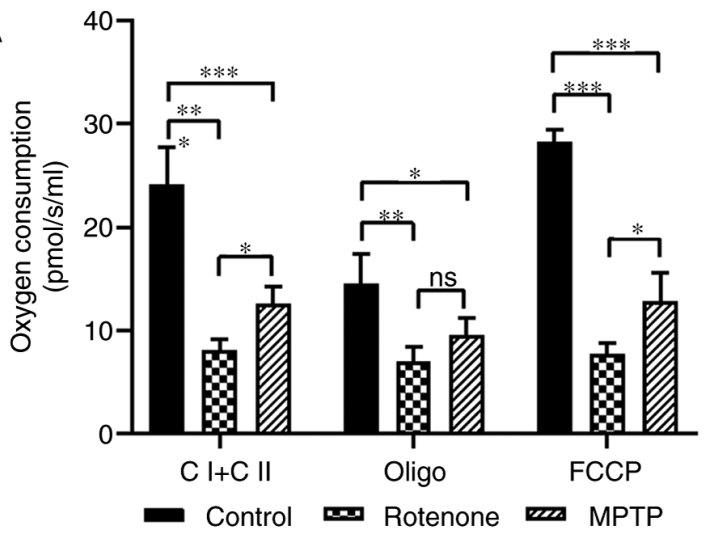

C

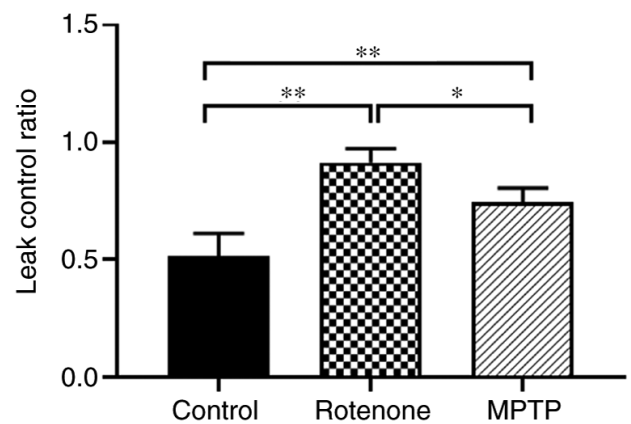

$\mathrm{B}$
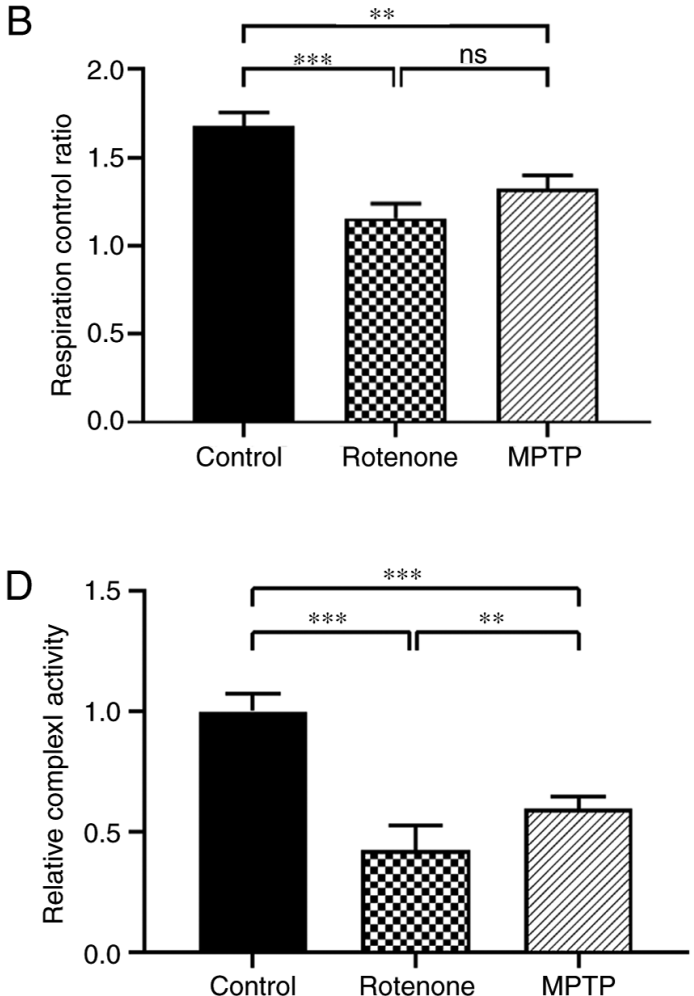

Figure 6. Oxygen consumption levels and complex I enzyme activity of mitochondria in the substantia nigra of rotenone and MPTP Parkinson's disease mouse models. (A) Mitochondrial oxygen consumption was measured via cell respiration, including total oxygen consumption of CI + CII, oxygen consumption after Oligo treatment and oxygen consumption after FCCP treatment. (B) Respiration control rate was defined as (CI + CII)/oligo. (C) Leak control rate was defined as oligo/FCCP. (D) Activity of complex I enzyme in each group was detected using a U-3900 spectrophotometer, with citrate synthase as the internal reference standard. ${ }^{*} \mathrm{P}<0.05,{ }^{* *} \mathrm{P}<0.01,{ }^{* * *} \mathrm{P}<0.001$. ns, no significant difference; MPTP, 1-methyl-4-phenyl-1,2,3,6-tetrahydropyridine; Oligo, oligomycin; FCCP, carbonyl cyanide-p-trifluoromethoxyphenylhydrazone; CI + CII, mitochondrial complexes I and II.

advantages and disadvantages (46). Although the pathogenic mechanisms underlying the genetic animal models of PD are precise, these models are complex, time-consuming and expensive to produce. Compared with the genetic animal models, neurotoxin-based PD animal models can reproduce the behavioral changes and pathological characteristics of PD (mainly sporadic PD), and the modeling protocols are more straightforward (47). The neurotoxin-based PD animal models reproduce the effects of toxic environmental factors associated with PD and have been widely used in model construction (48). For example, rotenone is widely used as a crop insecticide and fish poison in fish culture systems (49). Studies have demonstrated that long-term, low-concentration exposure to toxicants may be an environmental factor that can lead to PD (50-52). It also has been reported that MPTP is associated with PD caused by drug abuse (53). Therefore, the present study is of considerable practical importance for the neurotoxin-based PD models.

MPTP administration currently has become the most commonly used animal model of PD. There are three main models that use MPTP, including acute, subacute and chronic (54). The acute mouse model of PD has been created by injecting MPTP four times within $6 \mathrm{~h}(55,56)$. The subacute mouse PD model has been produced by injecting MPTP once a day for several days to several weeks (57). Finally, the chronic mouse model has been created by injecting MPTP and probenecid at 3.5-day intervals for 6 weeks. The acute model causes neuronal necrosis instead of apoptosis, which is not consistent with the actual disease (58). Zhang et al (59) revealed that it was challenging for the subacute MPTP administration to accurately simulate the motor dysfunction that occurs in patients with $\mathrm{PD}$ due to the compensatory response of the norepinephrine and DA systems, although it could produce damage to DA neurons and lead to the depletion of dopamine in the striatum. The chronic MPTP-induced mouse models exhibit a low mortality rate, chronic symptoms and stable pathology, which more accurately simulate the pathological process of PD (60). Therefore, MPTP (20 mg/kg) and probenecid were injected intraperitoneally twice a week for 6 weeks in the present study (61).

The chronic rotenone-induced mouse model also simulates the pathological and biochemical characteristics of patients with PD. The use of gavage administration effectively avoids severe injury that can result from repeated subcutaneous injections at the neck and back of the mouse (46). The chronic rotenone-induced mouse model is useful for studying changes that occur during PD and developing therapeutic drugs due to the low mortality rate and high success rate in model establishment $(62)$. Inden et al $(63,64)$ reported that the survival rate of rotenone-treated mice administered $30 \mathrm{mg} / \mathrm{kg}$ via gavage was $70 \%$ within 7 days and remained at $70 \%$ until 42 days. Therefore, rotenone is typically administered by gavage once a day for 6 weeks, using a dose of $30 \mathrm{mg} / \mathrm{kg}$.

In the present study, the results indicated that this dose selection, route and interval of administration of the neurotoxins resulted in the development of a stable animal model 
and minimized mortality. The survival rates of the rotenone and MPTP groups were 86.7 and $80 \%$, respectively, which provided feasible models to study PD. Since the results revealed no significant differences between the control group of $0.5 \% \mathrm{CMC}$ administered via gavage and the control group of $250 \mathrm{mg} / \mathrm{kg}$ probenecid administered via IP injection, the probenecid control group was not included in the present study.

The results demonstrated that the PD models induced by rotenone and MPTP produced significant alterations in behavior with no significant differences between the two groups. This observation suggested that chronic rotenone and MPTP exposure could accurately reproduce behavioral changes associated with PD. Although the differences were not significant, the motor function in the MPTP group demonstrated a trend to be more impaired compared with the rotenone group. Also, tremor was observed in the MPTP group but not in the rotenone group. These differences may be caused by a more severe damage of the SN-striatum pathway in the MPTP group, which has a more prominent effect on the regulation of movement by the basal ganglia (65).

When considering the pathological changes, the results indicated that the degeneration of DA neurons in the SNpc, dopamine depletion in the striatum and neuroinflammation in the SN induced by MPTP were significantly more severe compared with the rotenone group. It has been previously reported that both rotenone and MPTP are effective in inhibiting mitochondrial complex I, but they do so by acting on different sites of the complex (21). Rotenone inhibits complex I by binding to the coenzyme $\mathrm{Q}$ site competitively (66). However, increasing evidence supports the possibility that rotenone also reduces reactive oxygen species (ROS) production produced by the electron transport in the respiratory chain (67). Zawada et al (68) reported that the rotenone exposure of $\mathrm{MPP}^{+}$-treated $\mathrm{N} 27$ cell cultures suppressed $\mathrm{H}_{2} \mathrm{O}_{2}$ production by $50 \%$. Astrocytes convert MPTP into the neurotoxic molecule $\mathrm{MPP}^{+}$, which is taken up into DA neurons via the DAT (21). MPP ${ }^{+}$induces an initial wave of ROS production by binding to complex I and, subsequently, the second wave of ROS is mediated by extra-mitochondrial NADPH oxidase, which leads to multiple forms of cell damage (68). Furthermore, both rotenone and MPTP do not cause damage to DA neurons in the VTA (69). Due to the neuroinflammation in the SN induced by MPTP and rotenone, it may be necessary to detect the expression of soluble triggering receptor expressed on myeloid cells 2 (TREM2) in future studies, since soluble TREM2 in the cerebrospinal fluid (CSF) has been revealed to be a biomarker of neurodegeneration and glial activation in Alzheimer's disease (70). In several recent studies, soluble TREM2 in CSF has been reported as a potential biomarker of neuronal injury in Parkinson's disease (71-73).

It has been indicated that ATP-sensitive $\mathrm{K}^{+}\left(\mathrm{K}_{\text {ATP }}\right)$ channels are selectively activated in the SNpc but not in VTA, suggesting that the death of DA neurons in the SNpc may be associated with the activation of $\mathrm{K}_{\mathrm{ATP}}$ channels (74). $\mathrm{K}_{\mathrm{ATP}}$ channels are hetero-octamers formed with four regulatory sulphonyl urea receptor (SUR) subunits (SUR1, SUR2A or SUR2B) and four inwardly rectifying potassium channel subunits (Kir6.1 or Kir6.2). In a previous study on an MPTP-induced PD mouse model, SUR1 mRNA expression has been indicated to be two-fold higher in the SNpc DA neurons compared with the
VTA, suggesting that the selective upregulation of SUR1 leads to DA neuronal damage (75). In addition to DA neuronal loss, the presence of Lewy bodies in the remaining neurons represents a central pathological feature of PD (76). In the present study, chronic, low-dose rotenone treatment was more likely to lead to the accumulation of phosphorylated $\alpha$-synuclein in DA neurons, resulting in the formation of Lewy bodies, but MPTP treatment did not. This observation was consistent with several previous reports $(21,26)$.

$\alpha$-synuclein is a ubiquitously expressed small protein that is highly enriched in presynaptic nerve terminals, specifically on the highly curved membrane of synaptic vesicles (77). It has been demonstrated that rotenone accelerates the aggregation process of $\alpha$-synuclein monomers to form amyloid fibrils (78). Rotenone exposure also leads to the localization of $\alpha$-synuclein aggregates in the mitochondria and to decreased mitochondrial membrane potential, resulting in cell death (79). Rotenone may lead to $\alpha$-synuclein accumulation in the following two ways: i) Increasing the expression of $\alpha$-synuclein protein by enhancing the transcription of the $\alpha$-synuclein gene; and ii) preventing the degradation of $\alpha$-synuclein protein through the ubiquitin-proteasomal system and increasing phosphorylation of $\alpha$-synuclein at S129, which makes it more prone to aggregation (80). These observations suggested that the neuropathology of MPTP is more robust compared with rotenone, but MPTP does not reproduce all the pathological features of PD.

Mitochondria are indispensable for sustaining the high energy demand of neurons. It also is well known that mitochondria regulate programmed cell death, calcium homeostasis and numerous other important cellular processes (81). Mitochondrial dysfunction has been strongly implicated in the pathogenesis of PD. Plotegher and Duchen (82) have reported that a number of the causative or risk factor genes for PD are associated with mitochondrial quality-control pathways, ranging from mitochondrial proteins to proteins that regulate the endo-lysosomal function. Mitochondrial dysfunction also serves an important role in the pathogenesis of sporadic PD. Turner and Schapira (83) have reported decreased activity of respiratory chain complex I in the SN in brain tissue obtained from patients with PD at autopsy. Betarbet et al (84) observed complex I deficiencies in skeletal muscle and platelets from patients with PD. When mitochondrial complex I activity is decreased, a lower concentration of $\mathrm{H}_{2} \mathrm{O}_{2}$ can reduce the membrane potential of nerve fiber terminals. At the same time, blocking electronic respiratory chain transmission leads to increased free radical production and additional damage to complex I. Both rotenone and $\mathrm{MPP}^{+}$inhibit the activity of mitochondrial complex I (85), which impairs oxidative phosphorylation. Mitochondrial function studies have revealed a notable decrease in complex I activity and oxygen consumption in both PD models $(86,87)$. In the present study, rotenone exhibited a greater effect compared with MPTP. Abnormalities in mitochondrial function represented by the coupling level of electron transport and oxidative phosphorylation also were significantly aggravated in the Rotenone and MPTP groups compared with the control.

Rotenone exerts a stronger inhibition on mitochondrial complex I compared with MPTP, which may be attributed 
to the fact that rotenone can enter DA neurons directly and competitively bind to coenzyme Q to inhibit mitochondrial complex I without the interference of metabolism or the need for transporters. On the other hand, MPTP must be metabolized into $\mathrm{MPP}^{+}$in astrocytes and then enter DA neurons through the DAT. In addition, $\mathrm{MPP}^{+}$accumulates in mitochondria, synaptic vesicles and the cytoplasm in DA neurons. Thus, only a portion of the $\mathrm{MPP}^{+}$enters mitochondria and inhibits complex I $(21,88)$. Moreover, Plotegher and Duchen (82) have demonstrated that the inhibition of mitochondrial complex I activity by rotenone impairs lysosomal function and mitochondrial autophagy, resulting in an imbalance in the mitochondrial quality control mechanism.

In conclusion, the use of appropriate animal models will help researchers explore the causes and mechanisms of diseases and design more effective treatments. The present study indicated that the effects produced by the rotenoneand MPTP-induced chronic PD mouse models exhibited several important differences. While there was no significant difference in neurobehavior between the two models, the motor function of the animal models decreased significantly compared with the control group. Based on the current study, the MPTP model may be more suitable to study the loss of DA neurons, decreases in dopamine content and neuroinflammation in the SN. However, the chronic MPTP model had a higher mortality rate and lacked the most important neuropathological feature of PD, which is the formation of Lewy bodies. The rotenone model was more suited to study mitochondrial dysfunction (deficient complex I activity) and the formation of Lewy bodies in the SNpc. However, the degeneration of DA neurons in the SNpc, dopamine depletion in the striatum and neuroinflammation in the $\mathrm{SN}$ induced by rotenone were not as severe as in the MPTP model. Thus, the MPTP and rotenone PD models have advantages and disadvantages, therefore one or both should be selected based on the purpose of the study. The present study has provided a baseline for exploring the mechanism of the pathogenesis of PD, especially when seeking effective treatment methods for PD.

\section{Acknowledgements}

Not applicable.

\section{Funding}

The present study was supported by grants from the National Natural Science Foundation of China (grant no. 81971291), the Natural Science Foundation of Zhejiang Province (grant no. LY19C070001), the School of Laboratory Medicine and Life Sciences, Wenzhou Medical University (grant no. 437601607), the Beijing Friendship Hospital, Capital Medical University (grant no. YYZZ201920) and the Key Discipline of Zhejiang Province in Medical Technology (First Class, Category A).

\section{Availability of data and materials}

The datasets used and/or analyzed during the current study are available from the corresponding author on reasonable request.

\section{Authors' contributions}

JZ, BS and JY performed the experiments, analyzed the data and wrote the paper. ZC, ZL and NZ helped to performed the experiments and analyzed the data. LS and HL designed the research, analyzed the data and wrote and revised the paper. All authors confirm the authenticity of all the raw data. All authors have read and approved the final manuscript.

\section{Ethics approval and consent to participate}

The present study was approved by the Ethics Committee of Wenzhou Medical University (approval no. wydw2020-0840; Wenzhou, China).

\section{Patient consent for publication}

Not applicable.

\section{Competing interests}

The authors declare that they have no competing interests.

\section{References}

1. Alamri Y, Pitcher T and Anderson TJ: Variations in the patterns of prevalence and therapy in Australasian Parkinson's disease patients of different ethnicities. BMJ Neurol Open 2: e000033, 2020.

2. Jankovic J: Parkinson's disease: Clinical features and diagnosis J Neurol Neurosurg Psychiatry 79: 368-376, 2008.

3. Schneider SA and Obeso JA: Clinical and pathological features of Parkinson's disease. Curr Top Behav Neurosci 22: 205-220, 2015.

4. Schapira AH and Jenner P: Etiology and pathogenesis of Parkinson's disease. Mov Disord 26: 1049-1055, 2011.

5. Walter J, Bolognin S, Antony PMA, Nickels SL, Poovathingal SK, Salamanca L, Magni S, Perfeito R, Hoel F, Qing X, et al: Neural stem cells of Parkinson's disease patients exhibit aberrant mitochondrial morphology and functionality. Stem Cell Reports 12: 878-889, 2019.

6. Nakano M, Imamura H, Sasaoka N, Yamamoto M, Uemura N, Shudo T, Fuchigami T, Takahashi R and Kakizuka A: ATP maintenance via two types of ATP regulators mitigates pathological phenotypes in mouse models of Parkinson's disease. EBioMedicine 22: 225-241, 2017.

7. Wi S, Lee JW, Kim M, Park CH and Cho SR: An enriched environment ameliorates oxidative stress and olfactory dysfunction in Parkinson's disease with $\alpha$-Synucleinopathy. Cell Transplant 27: 831-839, 2018.

8. De Miranda BR and Greenamyre JT: Trichloroethylene, a ubiquitous environmental contaminant in the risk for Parkinson's disease. Environ Sci Process Impacts 22: 543-554, 2020.

9. Merhi R, Kalyn M, Zhu-Pawlowsky A and Ekker M: Loss of Parla function results in inactivity, olfactory impairment, and dopamine neuron loss in Zebrafish. Biomedicines 9: 205, 2021.

10. Fifel K and Videnovic A: Chronotherapies for Parkinson's disease. Prog Neurobiol 174: 16-27, 2019.

11. Mursaleen L, Noble B, Chan SHY, Somavarapu S and Zariwala MG: N-Acetylcysteine Nanocarriers protect against oxidative stress in a cellular model of Parkinson's disease. Antioxidants (Basel) 9: 600, 2020.

12. Wang B, Su CJ, Liu TT, Zhou Y, Feng Y, Huang Y, Liu X, Wang ZH, Chen LH, Luo WF and Liu T: The Neuroprotection of Low-Dose morphine in cellular and animal models of Parkinson's disease through ameliorating endoplasmic reticulum (ER) stress and activating autophagy. Front Mol Neurosci 11: 120, 2018.

13. Zhou L, Zhang Q, Zhang P, Sun L, Peng C, Yuan Z and Cheng J: C-Abl-Mediated Drp1 phosphorylation promotes oxidative stress-induced mitochondrial fragmentation and neuronal cell death. Cell Death Dis 8: e3117, 2017.

14. Bao XX, Ma HH, Ding H, Li WW and Zhu M: Preliminary optimization of a Chinese herbal medicine formula based on the neuroprotective effects in a rat model of rotenone-induced Parkinson's disease. J Integr Med 16: 290-296, 2018. 
15. Abdul-Latif R, Stupans I, Allahham A, Adhikari B and Thrimawithana T: Natural antioxidants in the management of Parkinson's disease: Review of evidence from cell line and animal models. J Integr Med 19: 300-310, 2021.

16. Sai Y, Chen J, Ye F, Zhao Y, Zou Z, Cao J and Dong Z: Dopamine release suppression dependent on an increase of intracellular $\mathrm{Ca}(2+)$ contributed to rotenone-induced neurotoxicity in $\mathrm{PC} 12$ Cells. J Toxicol Pathol 26: 149-157, 2013.

17. De Lazzari F, Bubacco L, Whitworth AJ and Bisaglia M: Superoxide radical dismutation as new therapeutic strategy in Parkinson's disease. Aging Dis 9: 716-728, 2018.

18. Qi Z, Miller GW and Voit EO: Rotenone and paraquat perturb dopamine metabolism: A computational analysis of pesticide toxicity. Toxicology 315: 92-101, 2014

19. Benskey MJ, Perez RG and Manfredsson FP: The Contribution of alpha synuclein to neuronal survival and function-implications for Parkinson's disease. J Neurochem 137: 331-359, 2016.

20. San Miguel M, Martin KL, Stone J and Johnstone DM: Photobiomodulation mitigates cerebrovascular leakage induced by the Parkinsonian neurotoxin MPTP. Biomolecules 9: 564, 2019.

21. Dauer W and Przedborski S: Parkinson's disease: Mechanisms and models. Neuron 39: 889-909, 2003.

22. Razali K, Othman N, Mohd Nasir MH, Doolaanea AA, Kumar J, Ibrahim WN, Mohamed Ibrahim N and Mohamed WM: The promise of the Zebrafish model for Parkinson's disease: Today's science and Tomorrow's treatment. Front Genet 12: 655550, 2021.

23. Bhurtel S, Katila N, Srivastav S, Neupane S and Choi DY: Mechanistic comparison between MPTP and rotenone neurotoxicity in mice. Neurotoxicology 71: 113-121, 2019.

24. MacArthur Clark JA and Sun D: Guidelines for the ethical review of laboratory animal welfare People's Republic of China national standard GB/T 35892-2018 [Issued 6 February 2018 Effective from 1 September 2018]. Animal Model Exp Med 3 . 103-113, 2020

25. Han X, Zhu J, Zhang X, Song Q, Ding J, Lu M, Sun S and Hu G: Plin4-dependent lipid droplets hamper neuronal mitophagy in the MPTP/p-induced mouse model of Parkinson's disease. Front Neurosci 12: 397, 2018

26. Zhou Q, Chen B, Wang X, Wu L, Yang Y, Cheng X, Hu Z, Cai X, Yang J, Sun $\mathrm{X}$, et al: Sulforaphane protects against rotenoneinduced neurotoxicity in vivo: Involvement of the mTOR, Nrf2, and autophagy pathways. Sci Rep 6: 32206, 2016.

27. Li T, Zhang W, Kang X, Yang R, Li R, Huang L, Chen J, Yang Q and Sun X: Salidroside protects dopaminergic neurons by regulating the mitochondrial MEF2D-ND6 pathway in the MPTP/MPP ${ }^{+}$-induced model of Parkinson's disease. J Neurochem 153: 276-289, 2020.

28. Ogrodnik M,Zhu Y,Langhi LGP, Tchkonia T, Krüger P,Fielder E, Victorelli S, Ruswhandi RA, Giorgadze N, Pirtskhalava T, et al: Obesity-induced cellular senescence drives anxiety and impairs neurogenesis. Cell Metab 29: 1061-1077.e8, 2019.

29. Wu CS, Chen H, Sun H, Zhu J, Jew CP, Wager-Miller J, Straiker A, Spencer C, Bradshaw H, Mackie K and Lu HC: GPR55, a $\mathrm{G}$-protein coupled receptor for lysophosphatidylinositol, plays a role in motor coordination. PLoS One 8: e60314, 2013.

30. Zhang W, Zhou M, Lu W, Gong J, Gao F, Li Y, Xu X, Lin Y, Zhang X, Ding L, et al: CNTNAP4 deficiency in dopaminergic neurons initiates Parkinsonian phenotypes. Theranostics 10 : 3000-3021, 2020.

31. Liu J, Liu W, Lu Y, Tian H, Duan C, Lu L, Gao G, Wu X, Wang $\mathrm{X}$ and Yang H: Piperlongumine restores the balance of autophagy and apoptosis by increasing BCL2 phosphorylation in rotenone-induced Parkinson disease models. Autophagy 14: 845-861, 2018

32. Zhao QY, Ge LH, Zhang K, Chen HF, Zhan XX, Yang Y, Dang QL, Zheng Y, Zhou HB, Lyu JX and Fang HZ: Assessment of mitochondrial function in metabolic dysfunction-associated fatty liver disease using obese mouse models. Zool Res 41: 539-551, 2020

33. Spinazzi M, Casarin A, Pertegato V, Salviati L and Angelini C: Assessment of mitochondrial respiratory chain enzymatic activities on tissues and cultured cells. Nat Protoc 7: 1235-1246, 2012

34. Liu J, Xu F, Nie Z and Shao L: Gut microbiota Approach-A new strategy to treat Parkinson's disease. Front Cell Infect Microbiol 10: 570658, 2020.

35. Shi W, Zhang Y, Zhao G, Wang S, Zhang G, Ma C, Cong B and $\mathrm{Li}$ Y: Dysregulation of dopaminergic regulatory factors TH, Nurrl, and Pitx3 in the ventral tegmental area associated with neuronal injury induced by chronic morphine dependence. Int J Mol Sci 20: 250,2019.
36. Domanskyi A, Alter H, Vogt MA, Gass P and Vinnikov IA Transcription factors foxal and foxa2 are required for adult dopamine neurons maintenance. Front Cell Neurosci 8: 275, 2014.

37. Wang XH, Lu G, Hu X, Tsang KS, Kwong WH, Wu FX, Meng HW, Jiang S, Liu SW, Ng HK, et al: Quantitative assessment of gait and neurochemical correlation in a classical murine model of Parkinson's disease. BMC Neurosci 13: 142, 2012.

38. Kelly E, Vyas P and Weber JT: Biochemical properties and neuroprotective effects of compounds in various species of berries. Molecules 23: 26, 2017.

39. Shin WH and Chung KC: Death-associated protein kinase 1 phosphorylates $\alpha$-Synuclein at ser129 and exacerbates rotenone-induced toxic aggregation of $\alpha$-Synuclein in dopaminergic SH-SY5Y Cells. Exp Neurobiol 29: 207-218, 2020.

40. Chen C, Wei YZ, He XM, Li DD, Wang GQ, Li JJ and Zhang F: Naringenin produces neuroprotection against LPS-induced dopamine neurotoxicity via the inhibition of microglial NLRP3 inflammasome activation. Front Immunol 10: 936, 2019.

41. Gangapuram M, Mazzio E, Eyunni S, Soliman KF and Redda KK Synthesis and biological evaluation of substituted $\mathrm{N}-[3-(1 \mathrm{H}-$ Pyrrol-1-yl)Methyl]-1,2,5,6-tetrahydropyridin-1-yl]Benzamide/ Benzene sulfonamides as anti-inflammatory agents. Arch Pharm (Weinheim) 347: 360-369, 2014.

42. Xu SF, Zhang YH, Wang S, Pang ZQ, Fan YG, Li JY, Wang ZY and Guo C: Lactoferrin ameliorates dopaminergic neurodegeneration and motor deficits in MPTP-treated mice. Redox Biol 21 101090, 2019

43. Barbon S, Rajendran S, Bertalot T, Piccione M, Gasparella M, Parnigotto PP, Di Liddo R and Conconi MT: Growth and differentiation of circulating stem cells after extensive ex vivo expansion. Tissue Eng Regen Med 18: 411-427, 2021

44. Fang Y, Zhao C, Xiang H, Zhao X and Zhong R: Melatonin inhibits formation of mitochondrial permeability transition pores and improves oxidative phosphorylation of frozen-thawed ram sperm. Front Endocrinol (Lausanne) 10: 896, 2019.

45. Gasperi V, Sibilano M, Savini I and Catani MV: Niacin in the central nervous system: An update of biological aspects and clinical applications. Int J Mol Sci 20: 974, 2019.

46. Chia SJ, Tan EK and Chao YX: Historical perspective: Models of Parkinson's disease. Int J Mol Sci 21: 2464, 2020.

47. Jagmag SA, Tripathi N, Shukla SD, Maiti S and Khurana S: Evaluation of models of Parkinson's disease. Front Neurosci 9: 503, 2015.

48. Goldman SM: Environmental toxins and Parkinson's disease. Annu Rev Pharmacol Toxicol 54: 141-164, 2014.

49. Zhang P, Qin D, Chen J and Zhang Z: Plants in the genus tephrosia: Valuable resources for botanical insecticides. Insects 11: 721,2020

50. Elbaz A and Moisan F: Update in the epidemiology of Parkinson's disease. Curr Opin Neurol 21: 454-460, 2008.

51. Wirdefeldt K, Adami HO, Cole P, Trichopoulos D and Mandel J: Epidemiology and etiology of Parkinson's disease: A review of the evidence. Eur J Epidemiol 26 (Suppl 1): S1-S58, 2011.

52. Das K, Ghosh M, Nag C, Nandy SP, Banerjee M, Datta M, Devi G and Chaterjee G: Role of familial, environmental and occupational factors in the development of Parkinson's disease. Neurodegener Dis 8: 345-351, 2011.

53. McKnight S and Hack N: Toxin-Induced Parkinsonism. Neurol Clin 38: 853-865, 2020.

54. Wang LY, Yu X, Li XX, Zhao YN, Wang CY, Wang ZY and He ZY: Catalpol exerts a neuroprotective effect in the MPTP mouse model of Parkinson's disease. Front Aging Neurosci 11: 316,2019

55. Tan Y, Xu Y, Cheng C, Zheng C, Zeng W, Wang J,Zhang X, Yang X, Wang J, Yang X, et al: LY354740 reduces extracellular glutamate concentration, inhibits phosphorylation of Fyn/NMDARs, and expression of PLK2/pS129 $\alpha$-Synuclein in mice treated with acute or sub-acute MPTP. Front Pharmacol 11: 183, 2020.

56. Jackson-Lewis V and Przedborski S: Protocol for the MPTP mouse model of Parkinson's disease. Nat Protoc 2: 141-151, 2007.

57. Li G, Luo W, Wang B, Qian C, Ye Y, Li Y and Zhang S: HMGA1 induction of MiR-103/107 forms a negative feedback loop to regulate autophagy in MPTP model of Parkinson's disease. Front Cell Neurosci 14: 620020, 2020.

58. Meredith GE and Rademacher DJ: MPTP mouse models of Parkinson's disease: An update. J Parkinsons Dis 1: 19-33, 2011.

59. Zhang QS, Heng Y, Mou Z, Huang JY, Yuan YH and Chen NH: Reassessment of Subacute MPTP-treated mice as animal model of Parkinson's disease. Acta Pharmacol Sin 38: 1317-1328, 2017. 
60. Mustapha M and Mat Taib CN: MPTP-induced mouse mode of Parkinson's disease: A promising direction of therapeutic strategies. Bosn J Basic Med Sci 21: 422-433, 2021.

61. Sung YH: Effects of treadmill exercise on hippocampal neurogenesis in an MPTP/Probenecid-induced Parkinson's disease mouse model. J Phys Ther Sci 27: 3203-3206, 2015.

62. Greenamyre JT, Betarbet R and Sherer TB: The rotenone model of Parkinson's disease: Genes, environment and mitochondria. Parkinsonism Relat Disord 9 (Suppl 2): S59-S64, 2003.

63. Inden M, Kitamura $Y$, Takeuchi H, Yanagida T, Takata $K$, Kobayashi Y, Taniguchi T, Yoshimoto K, Kaneko M, Okuma Y, et al: Neurodegeneration of mouse nigrostriatal dopaminergic system induced by repeated oral administration of rotenone is prevented by 4-Phenylbutyrate, a chemical chaperone. J Neurochem 101: $1491-1504,2007$.

64. Inden M, Kitamura Y, Abe M, Tamaki A, Takata K and Taniguchi T: Parkinsonian rotenone mouse model: Reevaluation of long-term administration of rotenone in $\mathrm{C} 57 \mathrm{bl} / 6$ mice. Biol Pharm Bull 34: 92-96, 2011.

65. Lieu CA and Subramanian T: The interhemispheric connections of the striatum: Implications for Parkinson's disease and drug-induced dyskinesias. Brain Res Bull 87: 1-9, 2012

66. Pravdic D, Hirata N, Barber L, Sedlic F, Bosnjak ZJ and Bienengraeber M: Complex I and ATP synthase mediate membrane depolarization and matrix acidification by isoflurane in mitochondria. Eur J Pharmacol 690: 149-157, 2012.

67. Murphy MP: How mitochondria produce reactive oxygen species. Biochem J 417: 1-13, 2009.

68. Zawada WM, Banninger GP, Thornton J, Marriott B, Cantu D, Rachubinski AL, Das M, Griffin WS and Jones SM: Generation of reactive oxygen species in 1-Methyl-4-Phenylpyridinium (MPP+) Treated dopaminergic neurons occurs as an NADPH oxidase-dependent two-wave cascade. J Neuroinflammation 8 : 129, 2011.

69. Brichta L and Greengard P: Molecular determinants of selective dopaminergic vulnerability in Parkinson's disease: An update. Front Neuroanat 8: 152, 2014.

70. Morenas-Rodríguez E, Alcolea D, Suárez-Calvet M, MuñozLlahuna L, Vilaplana E, Sala I, Subirana A, Querol-Vilaseca M Carmona-Iragui M, Illán-Gala I, et al: Different pattern of CSF glial markers between dementia with Lewy bodies and Alzheimer's disease. Sci Rep 9: 7803, 2019.

71. Peng G, Qiu J, Liu H, Zhou M, Huang S, Guo W, Lin Y, Chen X, $\mathrm{Li} \mathrm{Z,} \mathrm{Li} \mathrm{G,} \mathrm{et} \mathrm{al:} \mathrm{Analysis} \mathrm{of} \mathrm{cerebrospinal} \mathrm{fluid} \mathrm{soluble} \mathrm{TREM2}$ and polymorphisms in sporadic Parkinson's disease in a Chinese population. J Mol Neurosci 70: 294-301, 2020.

72. Wilson EN, Swarovski MS, Linortner P, Shahid M,Zuckerman AJ, Wang Q, Channappa D, Minhas PS, Mhatre SD, Plowey ED, et al: Soluble TREM2 is elevated in Parkinson's disease subgroups with increased CSF Tau. Brain 143: 932-943, 2020

73. Mo M, Tang Y, Wei L, Qiu J, Peng G, Lin Y, Zhou M, Dai W, Zhang Z, Chen X, et al: Soluble triggering receptor expressed on myeloid cells 2 from cerebrospinal fluid in sleep disorders related to Parkinson's disease. Front Aging Neurosci 13: 753210, 2021.
74. Liss B, Haeckel O, Wildmann J, Miki T, Seino S and Roeper J: K-ATP channels promote the differential degeneration of dopaminergic midbrain neurons. Nat Neurosci 8: 1742-1751, 2005.

75. Han SS, Jiao Q, Bi MX, Du XX and Jiang H: The expression of $\mathrm{K}_{\text {АтP }}$ channel subunits in alpha-synuclein-transfected MES23.5 cells. Ann Transl Med 6: 170, 2018.

76. Dickson DW: Neuropathology of Parkinson disease. Parkinsonism Relat Disord 46 (Suppl 1): S30-S33, 2018.

77. Burré J, Sharma M and Südhof TC: Cell biology and pathophysiology of $\alpha$-Synuclein. Cold Spring Harb Perspect Med 8: a024091, 2018.

78. Silva BA, Einarsdottir O, Fink AL and Uversky VN: Biophysical characterization of $\alpha$-Synuclein and rotenone interaction. Biomolecules 3: 703-732, 2013.

79. Wang Y, Shinoda Y, Cheng A, Kawahata I and Fukunaga K: Epidermal fatty acid-binding protein 5 (FABP5) Involvement in alpha-synuclein-induced mitochondrial injury under oxidative stress. Biomedicines 9: 2021

80. Cookson MR and van der Brug M: Cell systems and the toxic mechanism(s) of alpha-synuclein. Exp Neurol 209: 5-11, 2008

81. Rocha EM, De Miranda B and Sanders LH: Alpha-Synuclein: Pathology, mitochondrial dysfunction and neuroinflammation in Parkinson's disease. Neurobiol Dis 109: 249-257, 2018.

82. Plotegher N and Duchen MR: Crosstalk between lysosomes and mitochondria in Parkinson's disease. Front Cell Dev Biol 5: 110, 2017.

83. Turner $\mathrm{C}$ and Schapira AH: Mitochondrial dysfunction in neurodegenerative disorders and ageing. Adv Exp Med Biol 487: 229-251,2001.

84. Betarbet R, Sherer TB, MacKenzie G, Garcia-Osuna M, Panov AV and Greenamyre JT: Chronic systemic pesticide exposure reproduces features of Parkinson's disease. Nat Neurosci 3: 1301-1306, 2000

85. Udhayabanu T, Manole A, Rajeshwari M, Varalakshmi P, Houlden $\mathrm{H}$ and Ashokkumar B: Riboflavin responsive mitochondrial dysfunction in neurodegenerative diseases. J Clin Med 6: $52,2017$.

86. Ho PW, Ho JW, Liu HF, So DH, Tse ZH, Chan KH, Ramsden DB and Ho SL: Mitochondrial neuronal uncoupling proteins: A target for potential disease-modification in Parkinson's disease. Transl Neurodegener 1: 3, 2012.

87. Luo Y, Hoffer A, Hoffer B and Qi X: Mitochondria: A therapeutic target for Parkinson's disease? Int J Mol Sci 16: 20704-20730, 2015.

88. Patil DA, Patil VA, Bari SB, Surana SJ and Patil PO: Animal models for Parkinson's disease. CNS Neurol Disord Drug Targets 13: $1580-1594,2014$

This work is licensed under a Creative Commons Attribution-NonCommercial-NoDerivatives 4.0 International (CC BY-NC-ND 4.0) License. 